International Journal on Cybernetics \& Informatics (IJCI) Vol. 5, No. 4, August 2016

\title{
Performance Analsis of Clipping TechniQue FOR PAPR REDUCTION OF MB-OFDM UWB SIGNALS
}

\author{
PAPPU TEJASWI ${ }^{1}$ AND T.S.N MURTHY ${ }^{2}$ \\ ${ }^{1}$ Department of ECE, JNTUK-UCEV, Vizianagaram, Andhra Pradesh, India
}

\begin{abstract}
Multiband Orthogonal Frequency Division Multiplexing (MB-OFDM) is used as efficacious procedure for ultra-wideband (UWB) wireless communication applications, which divides the spectrum into various subbands, whose bandwidth is approximately 500MHz. Major arduousness in multiband-OFDM is, it have very large peak to average power ratio value which causes the signal to enter into dynamic region that consequence in the loss of orthogonal properties and results in the interference of the carrier signals which crops the amplifier saturation and finally limits the capacity of the system. Many PAPR amortize algorithms have reported in the survey and pre-coding is PAPR reduction which is inserted after modulation in the OFDM system. The Existing work presents the reduction of that value by different clipping techniques namely Classical-Clipping (CC), Heavy side-Clipping (HC), Deep-Clipping (DC) and Smooth-Clipping (SC) and their comparison analysis is done. Every clipping method is best at its own level.The proficiency of these strategies are evaluated in locutions of average power disparity, complete system decadence and PAPR reduction. Finally results show the MB OFDM yields better performance to reduce PAPR in effective way.
\end{abstract}

\section{KEYWORDS}

OFDM, PAPR, HPA, Clipping techniques, MB OFDM

\section{INTRODUCTION}

In present scenario, high data rate wireless links got a great importance, due the development of digital imaging and multimedia applications. Ultra Wide Band is emergent technology which facilitate that requirement of providing high data rate of $110 \mathrm{Mbps}$ at a distance of $10 \mathrm{~m}$ and 480 Mbps at a distance of $2 \mathrm{~m}[9]$.

This technology has raised a great influence in the wireless networking links because of its productive account in providing high data throughput, low power out sending, accurate ranging/localization and multipath immunity. MB-OFDM is more beneficial than the impulse radio' and other multiplexing based transmission. In this multiband multicarrier system the transmission symbol follows the high frequency hopping at pre-defined time -frequency interference (TFI) [9] pattern over the multiple bands. Timing and frequency synchronization is a defenceless issue in any Orthogonal FDM based systems. System performance perversion is caused due to the inter symbol interference and inter carrier interference which occurs due to time synchronization error. In MB-OFDM [5] system, frequency synchronicity is possible as source oscillator frequency offset generates different frequency carriers at distinct bands which provide high data transmission. It supports low intricacy, fast converging. 
International Journal on Cybernetics \& Informatics (IJCI) Vol. 5, No. 4, August 2016

MB-OFDM forms basis for all 4G wireless communication systems due to its huge capability in terms of number of subcarriers, high data rate in excess of $100 \mathrm{Mbps}$ and ubiquitous coverage with high mobility.

The introduction chapter consists of following parts:

In an MB-OFDM, more autonomous modulated sub-carriers are existing. Due to their presence, there is high Peak to average power ratio value of the system where peak value is ' $M$ ' times the average value due to addition of the same phase signals

The significant cons of a high Peak-Average Power Ratio are-

1. Complicacy increment in Analog -Digital convertors

2. Decline in efficiency of RF amplifier.

In this paper, MD-OFDM signals are generated and the High PAPR value is reduced using the clipping techniques, namely classical clipping, heavy side clipping, smooth clipping, deep clipping. The PAPR values are compared between the techniques. The average power, total degradation and BERVs SNR CCDF graphs are plotted

The remnant part of this particular paper is constituted following: section 2 introduced the Multiband-OFDM system model. Sub-section 3 deals with the clipping technique implementation for the PAPR abasement of MB-OFDM signals. In next section the simulation outputs are highlighted followed by the conclusion in the section 5 .

\section{Multiband OrThogonal Frequency Division Multiplexing (MB- OFDM) SYSTEM MODEL}

In this subsection, the basic MULTIBAND OFDM system is overviewed. . The federal communication commission (FCC) has declared the band width from $3.1 \mathrm{GHz}$ to $10.6 \mathrm{GHz}$ $(7.5 \mathrm{GHz})$ for ultra wide systems having spectral density of $-41.3 \mathrm{dBm} / \mathrm{MHz}[5] . \mathrm{UWB}$ system based on OFDM is combination of modulation technique with a multi- banding approach processed for efficient utilisation of spectrum having each band of 500Mhz approximately [6]. There is switching of sub bands after operating in one for some time. In each sub-band, OFDM modulation is used to transmit data symbols. In order to exploit the spectral diversity, the transmitted symbols are time interleaved across the sub-bands.

Multicarrier Communication involves splitting of the spectrum to give a number of signals over that frequency range i.e spectrum $7.5 \mathrm{GHz}$ into 14 bands each of $528 \mathrm{MHz}$ [9] [2].Each signal is modulated separately and transmitted over the channel and de-multiplexed at receiver side i.e demodulated and recombined to get the initial signal. This techniques implements OFDM scheme which is one of the multicarrier communication technique. Many advantages are posed by this procedure

- Transmitting power is same due to functioning over complete band-width

- Power consumption and cost are low due to processing of information in small bands

- Spectral pliability improvement 
International Journal on Cybernetics \& Informatics (IJCI) Vol. 5, No. 4, August 2016

\subsection{Representation OF Multiband-OrThogonal Frequency Division MULTIPLEXING (MB-OFDM) SIGNAL:}

The transmitted RF signal is associated to the base band signal as preceded

$$
S_{R F}(t)=\operatorname{Real}\left\{\sum_{i=0}^{M-1}\left(\begin{array}{c}
n \\
i
\end{array}\right) s_{i}\left(t-k T_{S Y S}\right) e^{J 2 \pi f_{\text {imod } 6}}\right\}
$$

Where Real (.) delineate complex variable real value. $s_{i}(\mathrm{t})$ is the base band signal representing the $i^{\text {th }}$ OFDM symbol occupying a symbol interval of length $T_{S Y S}$ and $M$ in the number of OFDM symbol transmitted the carrier frequency or band that the $\mathrm{i}^{\text {th }}$ OFDM symbol is transmitted over is denoted as $f_{i}$ range over 3 frequencies assigned to the band group that the system is operating in those frequencies are organised into sequence of length 6 ,called time-frequency code(TFC).Location of the $\mathrm{i}^{\text {th }}$ symbol in the packet decides it's exact location All the OFDM symbol $s_{i}(\mathrm{t})$ is obtained with a certain set of Coefficients $C_{n}$ which are applied IFFT operation. the coefficients are defined as either data, pilot or training symbols.

$$
s_{i}(\mathrm{t})=\left\{\begin{array}{cc}
\sum_{-N_{S T / 2}}^{N_{S T / 2}} C_{n} e^{J 2 \pi n \Delta \mathrm{f}(\mathrm{t})} & , \mathbf{t} €\left[\mathbf{0}, T_{F F T}\right] \\
\mathbf{0} & , \mathbf{t} €\left[T_{F F T}, T_{F F T}+T_{Z p}\right]
\end{array}\right.
$$

where $\Delta \mathrm{f}$ represents carrier frequency and $N_{S T}$ represents the subcarrier frequency spacing and number of total subcarrier users respectively[8].The final waveform have time period of $T_{F F T}=$ $1 \Delta \mathrm{f}$ zero padding $\mathrm{T}_{\mathrm{zp}}$ is done to provide guard interval between different sub-bands as well as to avoid the multipath effect.

\section{Peak To Average Ratio Reduction Of Multiband Orthogonal Frequency Division Multiplexing (Mb-OfdM) Signals Using ClipPING TeChNIQUeS}

In this section, the description of PAPR [4] and PAPR reduction done by clipping technique is given. Clipping techniques mainly focused on classical clipping, deep clipping, heavy side clipping and smooth clipping.

\subsection{Peak To Average Power Ratio (PAPR)}

High date rate communication is very adverse task in wireless communication. With the ever growing demand of wireless communication in this generation various modulation techniques are introduced. Multi band multi carrier frequency division multiplexing is one of prominent technology amongst. There are different techniques to diminish PAPR of OFDM. It can be divided into two types. They are signal scrambling techniques and signal distortion techniques. The complex data block for the OFDM signal to be transmitted is given by

$$
y(t)=\frac{(1)}{\sqrt{M}} \sum_{k=1}^{M-1} Y_{k} e^{j 2 \pi \mathrm{fkt}}
$$

Where $\mathrm{M}$ is number of sub-carriers $Y_{k}$ is the $\mathrm{k}^{\text {th }}$ component of symbol $\mathrm{Y}, \mathrm{f}_{\mathrm{k}}$ frequency of the $\mathrm{k}^{\text {th }}$ subcarrier.

$$
P A P R=\frac{\text { peak instantanous power }}{\text { avearge power }}=\frac{\max |y(t)|^{2}}{E\left[|y(t)|^{2}\right]}
$$


Where E [·] states the expected value [4].

\subsection{CliPPING TEChNIQUeS}

Among, the all the methods of PAPR reduction, clipping technique [4] is simplest .In this method, high peak values of the multicarrier signal are clipped before it passes through the power amplifier. This is done by clipper which restrict the signal envelop to the fore determined threshold level known as clipping level[10].this technique may cause distortion which emanate in-band and out-band disparity, which is solved using filter. The implementation of the clipping is demoed through the following diagram

Figure 1 explains the multicarrier MB-OFDM transmitter which consists of mainly clipping equipments and filter. Depending on the type of the clipping technique used for PAPR reduction, the behaviour of clipper varies, which is given by the function n.f(.)The filter consists of FFT and IFFT pair where FFT transmogrify $\hat{Y}_{n}$ to $\hat{Y}_{k}$ i.e from frequency to time domain. The in-band components of $Y^{\sim}$ is passed without any change where as the out-of-band components are set to zero, which is given as

$$
X_{k}=\left\{\begin{array}{l}
Y_{k}, \quad \overline{k \in} \text { In } \\
0, \quad k \in \text { Out }
\end{array}\right.
$$

Where as In is in-band, Out is out-of-band components of Y.Y $\mathrm{Y}_{\mathrm{n}}$ is the MDOFDM symbol, $\hat{\mathrm{Y}}_{\mathrm{n}}$ is the clipping symbol $\mathrm{Xn}^{\wedge}$ is the clipped output and $\mathrm{f}_{\text {car }}$ is carrier frequency.

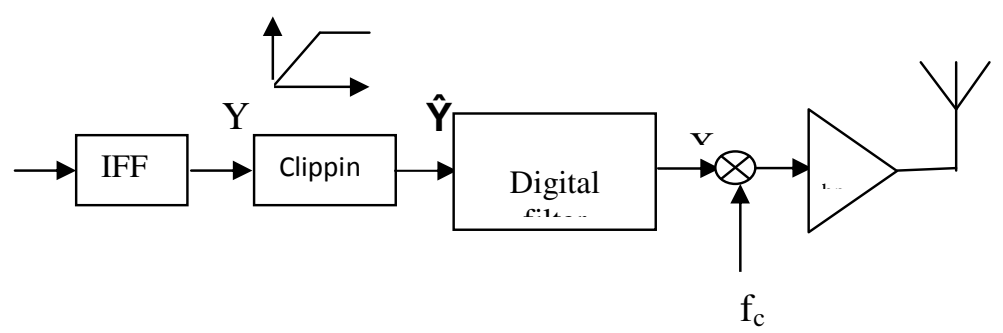

Figure 1: multicarrier (MB-OFDM) transmitter

\section{VARIOUS ClipPING TECHNIQUeS IMPLEMENTED For PAPR REDUCTION}

In this paper only four clipping techniques are highlighted: Classical clipping, Heavy side clipping, Deep Clipping and Smooth Clipping[4] whose the functions of clipping are depicted in figure 2. The discrete-time OFDM signal $Y_{n}$ is rewritten into polar coordinates gives $Y_{n}=p_{n}$ $\mathrm{e}^{\mathrm{j} \theta \mathrm{n}}$, where $\mathrm{p}_{\mathrm{n}}$ implied signal amplitude $\mathrm{Y}_{\mathrm{n}}$ and $\Theta_{\mathrm{n}}$ is phase .The clipped signal $\mathrm{Y} \sim \mathrm{n}$ is expressed as

$$
\hat{Y}_{n}=f\left(p_{n}\right) e^{j \omega n}
$$

\subsubsection{Classical ClipPing (CC) TechniQue}

The most popular PAPR reduction technique proposed in the literature known as far is CLASSICAL CLIPPING (CC)[4]effects PSD and MB-OFDM performance This function is given as follows and depicted in fig2(a) 


$$
f(p)= \begin{cases}S & , p>S \\ p & , p \leq S\end{cases}
$$

\subsubsection{HeAvy Side ClipPING (HC) TECHNIQUE}

Where $\mathrm{S}$ is the threshold amplitude considered.

It is also called hard clipping. The communication system execution is improved by using Heavy side clipping as baseband non linear transformation technique[4] this is give by the following function, depicted in fig2(b)

$$
\mathrm{f}(\mathrm{p})=\mathrm{S} \text { ? } \mathrm{p} \geq 0
$$

\subsubsection{DEEP CLIPPING (DC) TECHNIQUE}

Deep clipping (DC)[4] is proposed to solve the problem of peaks re-growth caused due to out-of band filtering. Here the function is altered to avoid high amplitude peaks. Depth factor is parameter that is nait to control the clipping depth. This technique is given as below and paraded infig2(c)

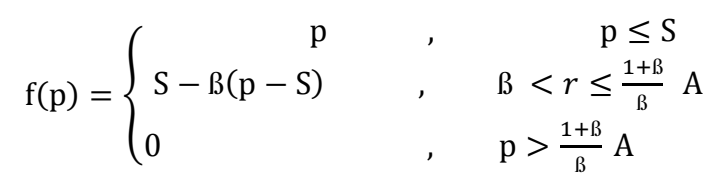

Where $B$ is depth factor.
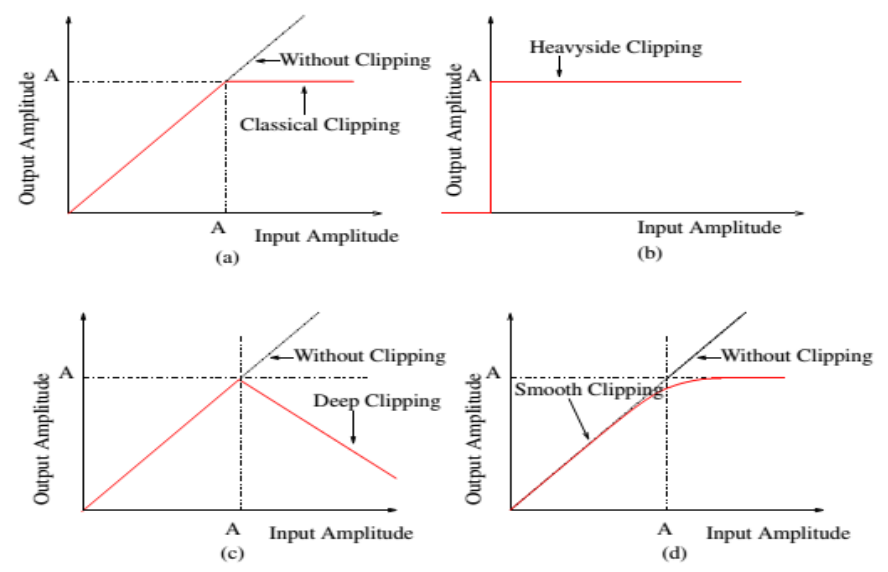

Figure 2.Different Clipping techniques

\subsubsection{SMOOTh CLIPPING (SC) TECHNIQUe}

MB-OFDM peak-average power is diminished using this method. The function is given as follows and the characteristic graph is shown in 2(d)

$$
f(p)=\left\{\begin{array}{cl}
p-\frac{1}{r} p^{3} & , p \leq \frac{3}{2} S \\
S & , p>\frac{3}{2} S
\end{array} \quad \text {, where } r=\frac{27}{4} S^{2}\right.
$$




\section{EXECUTION RESULT}

The system performances are calculated considering the factors like clipping ratio(CR)[4] and input back off(InBO).

$$
\begin{aligned}
& \text { ClippingRatio }=20 \log 10 \frac{\mathrm{s}}{\sqrt{K}}[\mathrm{~dB}] \\
& \text { InBO }=-10 \log 10 \frac{\left(\text { Exp }\left|C_{\text {in }} \cdot C_{\text {in }} \cdot *\right|\right)}{K_{\text {satar }}} \mathrm{dB}
\end{aligned}
$$

Here $\mathrm{K}$ is knee factor which decides the smoothness, $K_{\text {satar }}$ is signal saturated power.

\subsection{ObServation Parameters}

The peak-average power ratio is computed by taking the instantaneous power normalised with the expectation of square of average power the peak power is calculated based on complementary cumulative distribution function [1]

$$
\operatorname{CompCumDisFun}(\theta)=\operatorname{Prob}\{\text { Peak } \geq \theta\}
$$

Which means probability of samples that surpass the instantaneous power $(\Theta)$

The three parameters which are considered for the system execution in this paper are

- $\Delta$ Ppapr which marks the peak to average power reduction performance[4]that is described as

$$
\Delta \text { Ppapr }=P_{[\text {Clipped }]}-P_{[\text {Not Clipped }]} \mathrm{d} ß
$$

Where $P_{[\text {Clipped }]}$ gives the value of CCDF when clipping is exercised

- Total system degradation[4] states the transmission of the system which is defines as

$$
\text { Toatl sys Deg }=\Delta \mathrm{ENR}+\mathrm{InBO}
$$

Where $\triangle \mathrm{ENR}$ is degraded energy per bit and InBO is input back off value

- Third factor, Average peak power difference shown by $\Delta \mathrm{P}$

$$
\Delta \mathrm{P}=A v g_{\text {[clippedvalue] }}-A v g_{[\text {[noclippedvalue] }}
$$

Where $\operatorname{Avg}_{\text {[clipped value] }}$ is the mean power of clipped signal and $A v g_{\text {[no clipped value] }}$ is the value obtained when no clipping is done.

\begin{tabular}{|l|l|}
\hline Modulation scheme & QPSK \\
\hline Subcarrier number & $\mathrm{M}=128$ \\
\hline Oversampling factor[4] & $\mathrm{L}=4$ \\
\hline Channel [4] & AWGN \\
\hline
\end{tabular}

TABLE 1: Modelling measurements 
International Journal on Cybernetics \& Informatics (IJCI) Vol. 5, No. 4, August 2016

Figure 4:original MB-OFDM sequence, peak sequence and outputs of different clipping techniques .MB-OFDM sequence which demonstrates that more number or users using the band (i.e 1500's in number)while the other subplots depicts the amplitude clipping by the different clipping techniques[4].

Figure 5: Total system degradation Vs input back off (IBO) This figure depicts the system degradation performance when the different clipping techniques are applied at the Bit Error rate of $10^{-2}$. classical clipping and deep clipping have well-nigh the same result in this case, where as the smooth clipping technique gave more power degradation compared with the remaining two the minimum system degradation value for classical and deep clipping is around $6.5 \mathrm{~dB}$ but for smooth clipping it is around 7.5dB.As per this parameter considered final line ,this deep clipping is good with minimum loss of $6.43 \mathrm{~dB}$ (classical clipping $=6.54 \mathrm{~dB}$ )

Figure 6:different clipping ratios[4] and their effect on the Peak to average power ratio reduction performance for deep clipping technique This figure provides the performance of the PAPR reduction by deep clipping technique at different clipping ratios (for convince only three values are considered).the value of PAPR remains almost constant for the clipping ratio values < $3 \mathrm{~dB}$, where as for the clipping ratio values $>3 \mathrm{~dB}$ the PAPR value varies .For the clipping depth factor (CDF) [4] value greater than 0.5,the graph shows nigh same value of PAPR (whatever may be the clipping ratio value).

Figure 7: clipping ratio effect on the PAPR reduction gain. This figure exemplify the PAPR Reduction gain performance[4] for different clipping ratio values for different clipping techniques .PAPR reduction gain value for classical clipping is next to smooth clipping. There is a linear decrease in the gain value from clipping ratio=4dB.deep clipping shows good performance compared with classical and smooth clipping techniques the gain value decreases from $\mathrm{CR}=8 \mathrm{~dB}$ for deep clipping. After $10 \mathrm{~dB}$ of clipping ratio value the reduction gain becomes zero and there will be no more reduction.

Figure 8: clipping techniques-classical clipping, deep clipping, smooth clipping and heavy side clipping analysis through average power deed. This figure shows the different clipping techniques performance based on average power [1]. Transmission quality is affected by transmission signal average power .so the technique which has avg power $=0 \mathrm{~dB}$ is considered to be the best. There is decrease in the average power with clipping ratio factor increase in case of deep, classical and smooth techniques from clipping ratio factor $=4 \mathrm{~dB}$. Heavy side clipping have no effect by clipping ratio on PAPR reduction gain so the avg power=0dB.so it is considered as best at CR[4] factor $=4 \mathrm{~dB}$ to $6 \mathrm{~dB}$.

Figure 9: peak to average power ratio reduction by clipping techniques. This figure demonstrate the different clipping techniques performance on PAPR reduction the value of PAPR without applying any clipping technique is obtained as $29.84 \mathrm{~dB}$. while classical clipping gave nearly half of the value $(14.29 \mathrm{~dB})$.smooth and deep clipping techniques gave similar outputs around $8 \mathrm{~dB}$. In particular smooth clip technique gave the best result of $7.83 \mathrm{~dB}$.

Figure 10: Bit error rate verses energy per bit [4] of different clipping methods. This shows that the Smooth clipping is more advantageous compared to the deep clipping and the classical clipping technique.BER Vs Eb/NO graph here shows that the clipping techniques showed good output than without clipping 
International Journal on Cybernetics \& Informatics (IJCI) Vol. 5, No. 4, August 2016

\section{OUTPUTS}

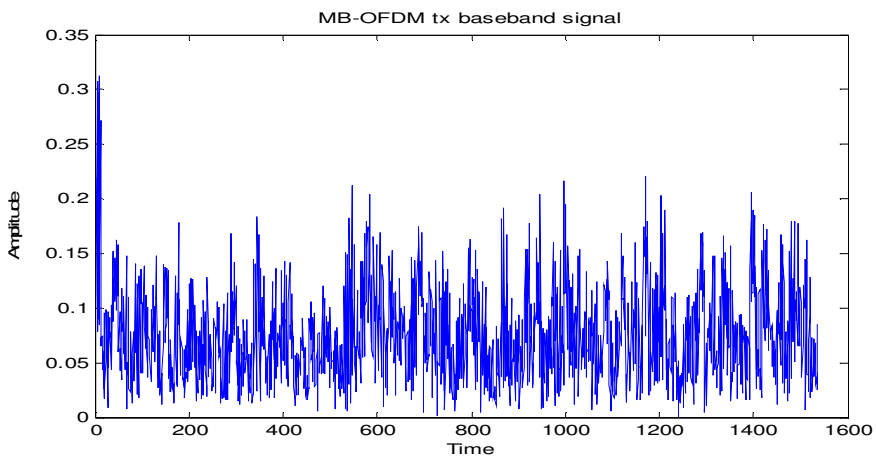

Figure 3:MB-OFDM Transmitted Baseband Signal
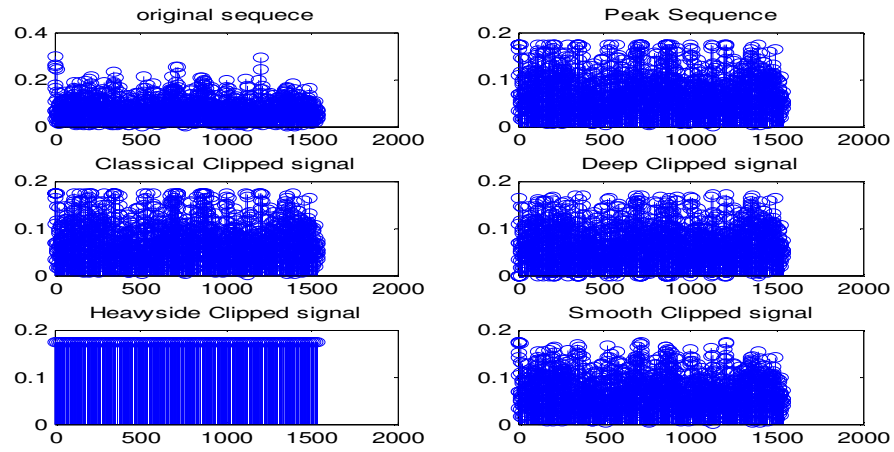

Figure 4: Original MB-OFDM Sequence, Peak Sequence And Outputs Of Different Clipping Techniques

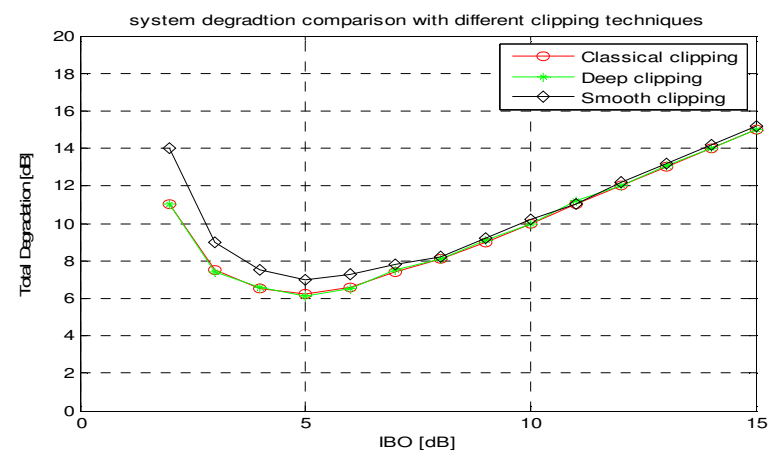

Figure 5: Total System Degradation Vs Input Back Off(IBO) 
International Journal on Cybernetics \& Informatics (IJCI) Vol. 5, No. 4, August 2016

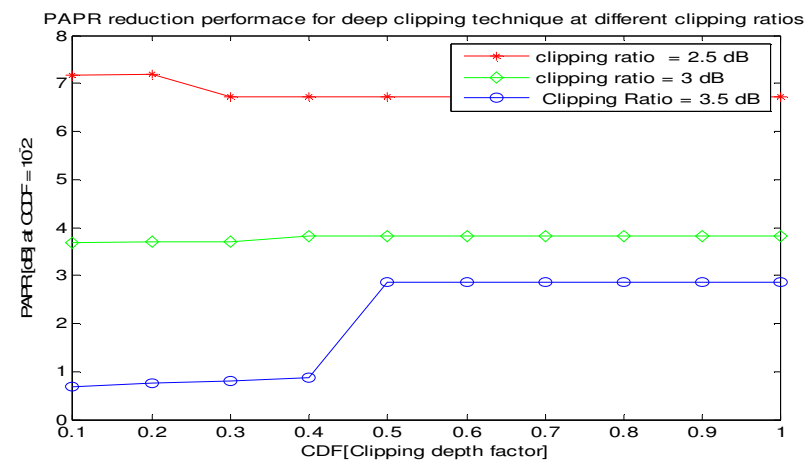

Figure 6: Different Clipping Ratios [4] And Their Effect On The PAPR Reduction Performance For Deep Clipping Technique.

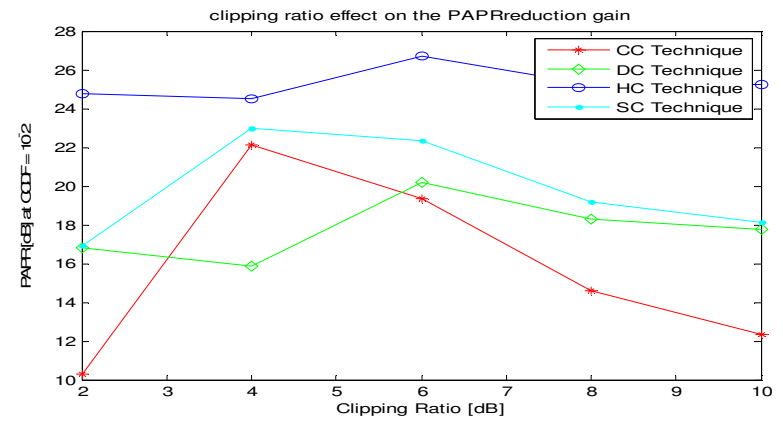

Figure 7: Clipping Ratio Effect On The PAPR Reduction Gain.

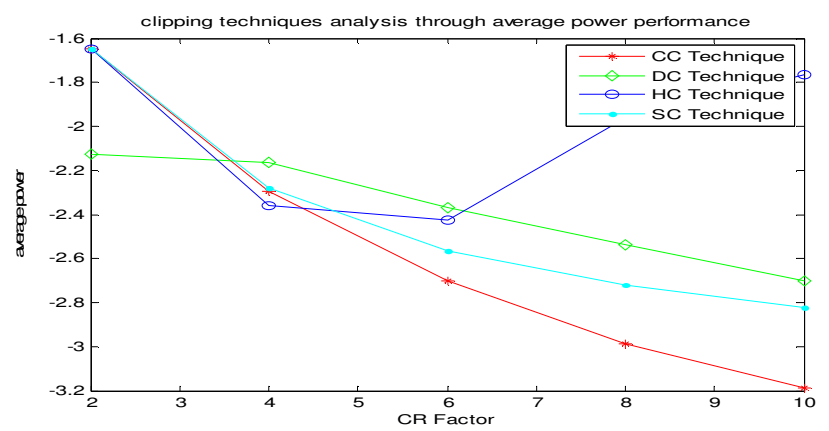

Figure 8: Clipping Techniques Analysis Through Average Power Deed.

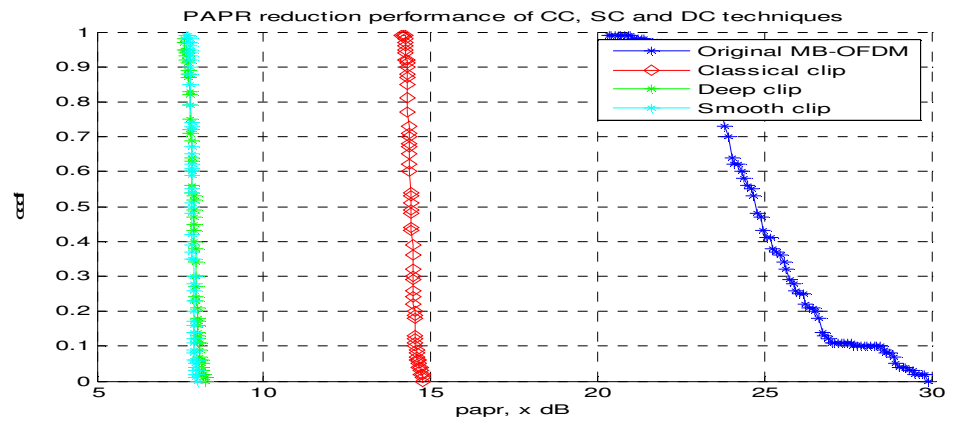

Figure 9: Peak To Average Power Ratio Reduction By Clipping Technique 


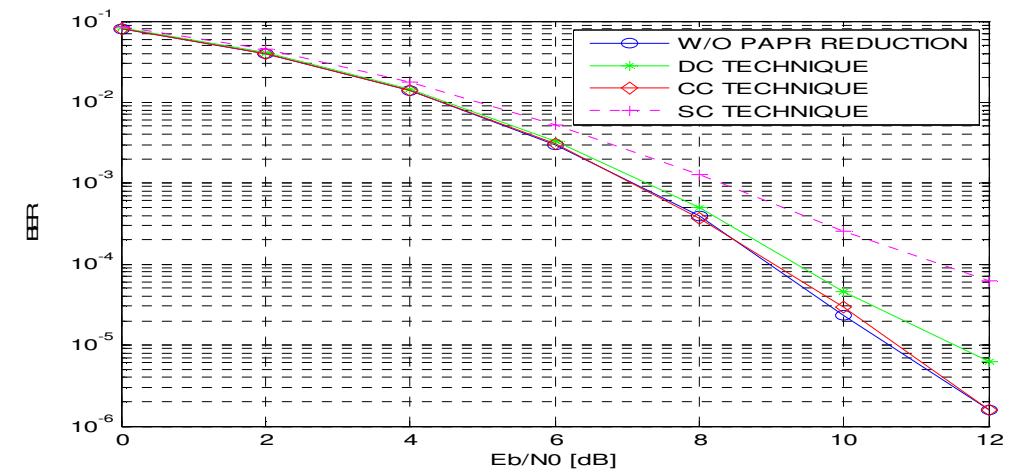

Figure 10: Bit Error Rate Verses Energy Per Bit [4] Of Different Clipping Methods

\section{Conclusion}

Although tremendous progress has been made in the past decade to reduce the PAPR in OFDM system but still it is consider as an area of concern in the wireless communication scenario. MBOFDM[5] for UWB technology[6] flaunts a noumenon technacy for prominent set of short range (distance) and high performance application. But this scheme causes high Peak-to-Average Power Ratio (PAPR) resulting in the saturation of High Power Amplifier.

In this work a genuine weighted scheme is forth put to minimise the PAPR [1]in an efficient way without any distortion and successfully remove the respective weights at the receiver end. The reduction of PAPR in multicarrier system mainly relies on condition that time consumes at the Transmitter end should be same as at the receiver end.

In this paper a comparative analysis between different clipping methods[4] Classical-Clipping (CC), Heavy side-Clipping (HC), Deep-Clipping (DC) and Smooth-Clipping (SC)in terms of total system degradation, Peak to average power ratio(PAPR) reduction and average power performance is accomplished. Heavy side clipping have the worst results amongst the four because of its high Bit error rate (BER) degradation. After intense research and experiments finally DC clipping technique is best resultant among four clipping techniques because it gives best result in considered parameters- total degradation, average system power and PAPR limiting .When the depth factor of deep clipping is equivalent to zero Deep and classical clipping(CC)techniques are identical . It is envisioned to define the wireless links engross the communication rate to the intense.

\section{REFERENCES}

[1] T. Jiang and Y. Wu, "An overview: Peak-to-average power ratio reduction techniques for OFDM

[2] S. H. Han and J. H. Lee, "An overview of peak-to-average power ratio reduction techniques for multicarrier transmission,” IEEE Trans. Wireless Commun., vol. 12, no. 2, pp. 56-65, Apr. 2005.

[3] R.W.Bäuml, R.F.H.Fischer and J.B.Huber "Reducing the peak-to average power ratio of multicarrier modulation by selected mapping," IEEE Electron. Lett., vol. 32, no. 22, pp. 2056-2057, Oct. 1996.

[4] Desire Guel :analysis and comparison of clipping techniques for OFDM Peak -to-average power ratio reduction"200916th for ofdm peak-to-average power ratio reduction"2009 16th international conference on digital signal processing, 07/2009

[5] W. Pam Siriwongpairat, Weifeng Su, Masoud Olfat, and K. J. Ray Liu.” Multiband-OFDM MIMO Coding Framework for UWB Communication Systems", IEEE TRANSACTIONS ON SIGNAL PROCESSING, VOL. 54, NO. 1, JANUARY 2006 
International Journal on Cybernetics \& Informatics (IJCI) Vol. 5, No. 4, August 2016

[6] Chia-Chin Chong, Fujio Watanabe, and Hiroshi Inamura“Potential of UWB Technology for the Next Generation Wireless Communications”, 2006 IEEE Ninth International Symposium on Spread Spectrum Techniques and Applications

[7] Anuj Batra, et al., -Design of a Multiband OFDM System for Realistic UWB Channel Environments \|, IEEE TRANSACTIONS ON MICROWAVE THEORY AND TECHNIQUES, VOL. 52, NO. 9, SEPTEMBER 2004.

[8] Sajjad Hussain, Yves Louët Peak to Average Power Ratio Reduction for Multi-band OFDM System using Tone Reservation IEEE Trans. on Wireless Communications, pp. : June 2010

[9] Debarati Sen. "Frequency Offset Estimation by Multi-Band Averaging Method: A New Approach for MB-OFDM Based Ultra- Wideband Communication System", Wireless Personal Communications, 08/05/2009

[10] W. Aziz, E. Ahmed, G. Abbas, S. Saleem and Q. Islam” PAPR Reduction in OFDM using Clipping and Filtering" World Applied Sciences Journal 18 (11): 1495-1500, 2012 W. Aziz, Department of Electrical Engineering, Institute of Space Technology, Islamabad Pakistan 149

\section{AUTHOR}

T.S.N.Murthy

Assistant Professor,

Dept. of ECE ,JNTUK-UVCE, Vizianagaram.

LMISTE, MIE, MIETE,

Member of IET,

Senior Member of IEEE

Secretary, IEEE Vizag Bay subsection,

P.Tejaswi

Completed B.Tech.(ECE)degree from BITS-vizag,

AndhraPradesh.

Perusing M.Tech. (Systems \& Signal Processing),

JNTUK-UCEV,Vizianagaram. 